\title{
Critical behaviour of the three-dimensional gonihedric Ising Model
}

\author{
E.N.M. Cirillo ${ }^{a}$, G. Gonnella ${ }^{a}$, A. Pelizzola ${ }^{b}$ \\ apipartimento di Fisica dell'Università degli Studi di Bari and Istituto Nazionale di Fisica Nucleare, \\ Sezione di Bari via G. Amendola 173, I-70126 Bari, Italy \\ ${ }^{\mathrm{b}}$ Dipartimento di Fisica del Politecnico di Torino and Istituto Nazionale di Fisica per la Materia, c. \\ Duca degli Abruzzi 24, 10129 Torino, Italy
}

\begin{abstract}
We use the cluster variation method (CVM) and Monte Carlo simulations to investigate the phase structure of the 3d gonihedric Ising actions defined by Savvidy and Wegner. This model corresponds to the usual threedimensional cubic Ising model with nearest, next to the nearest, and plaquette interactions in the region with degenerate lamellar ground states. The picture of the phase diagram given by the CVM is in good agreement with the results of Monte Carlo simulations, and it is shown that the gonihedric model is in the same universality class of the ordinary three-dimensional Ising model.
\end{abstract}

The 3d Ising model with interactions extended to next-to-the-nearest neighbouring $(\mathrm{NNN})$ sites and 4-spin plaquette interaction has a very rich phase diagram relevant for the description of physical systems of interacting interfaces [1].2]. In this paper we discuss the critical behaviour of this model in a particular region of parameters first explicitly considered in [3] in relation with string theory.

Ising variables can describe surface configurations in a simple way to explain: for any spin configuration there is on the dual lattice a set of closed interfaces separating the domains of spins with different sign. If $\beta_{A}, \beta_{C}, \beta_{I}$ respectively are the energy cost for an unit area, two connected plaquettes forming a right angle, and four plaquettes intersecting at a common link, the corresponding Ising model is given by

$$
\begin{aligned}
-H & =J_{1} \sum_{\langle i j\rangle} \sigma_{i} \sigma_{j}+J_{2} \sum_{\langle\langle i, j\rangle\rangle} \sigma_{i} \sigma_{j} \\
& +J_{4} \sum_{[i, j, k, l]} \sigma_{i} \sigma_{j} \sigma_{k} \sigma_{l} .
\end{aligned}
$$

with $J_{1}=\beta_{C}+\left(\beta_{A}+\beta_{I}\right) / 2, J_{2}=-\left(2 \beta_{C}+\right.$ $\left.\beta_{I}\right) / 8, J_{4}=\left(2 \beta_{C}-\beta_{I}\right) / 8$ [4]. In eq. (1]) the three sums respectively are on the nearest neighbours, the NNN spins and the plaquettes of the cubic lattice. The gonihedric model is the model (11) with $\beta_{A}=0\left(J_{2} / J_{1}=-1 / 4\right)$ and $\beta_{C}=1$. The choice $\beta_{A}=0$ means that the amount of surfaces in the system is not controlled by any ex- ternal parameter, while $\beta_{C}=1$ gives a preference to flat surfaces. The parametrization $J_{1}=$ $2 k, J_{2}=-k / 2, J_{4}=(1-k) / 2$ is often used; the parameter $k=\beta_{I} / 4+1 / 2$ can interpolate between completely non-interacting $(k=1 / 2)$ and self-avoiding surfaces $(k=\infty)$.

The ground state degeneracy is the characteristic of the gonihedric Ising model: all the possible sequences of "+" and "-" spin planes (for $\left.J_{4} / J_{1}>-1 / 4\right)$ have the same minimum energy. The situation changes for $\beta_{A} \neq 0$ : a positive $\beta_{A}$ selects the ferromagnetic ground state (no interface allowed) while at negative $\beta_{A}$ the ground state is a sequence of planes with spins of alternate sign (maximum amount of flat interfaces).

The finite temperature behaviour of the model can be easily studied by means of the mean field approximation, which predicts that the ground state symmetry is preserved at finite temperature (that is, the first order line between lamellar and ferromagnetic phases in Fig. 1 would be strictly vertical, independent of temperature) and, at least for $k>3 / 4$, the ferromagnetic and lamellar phases are separated from the disordered one by two second order lines (both turning first order for $k<3 / 4$ ) which meet in a bicritical point at $J_{2} / J_{1}=-1 / 4$.

Recently, several studies based on high temperature expansions [5] and Monte Carlo simu- 
lations [6] addressed the issue of the universality class of the gonihedric model (the existence of a phase with long-range order has been analytically proved in 7 for the case $J_{4}=0$ ), formulating the conjecture that the model might be in the Onsager (i.e. two-dimensional Ising) universality class. Further, longer simulations [8], however, found estimates for critical exponents which were seriously incompatible with both the conjecture and the previous numerical estimates.

We have studied the gonihedric model at finite temperature by means of the cluster variation method (CVM) [9, 10], which is a powerful generalization of the mean field approximation [11]. Our results show that the groundstate degeneracy along the line $J_{2} / J_{1}=-1 / 4$ is broken at finite temperatures and the ferromagnetic phase is stable along this line. In Fig. 1 we show the phase diagram in the space $\left(1 /\left(\beta J_{1}\right), J_{2} / J_{1}\left(J_{4}=0\right)\left(\beta=\left(k_{B} T\right)^{-1}\right)\right.$ as calculated in the cube approximation of the CVM. We see that the ferromagnetic-lamellar transition line bends at finite temperatures towards values of $J_{2} / J_{1}$ lower than $-1 / 4$; this bending has been confirmed by low-temperature expansion results [9. 12] and is very important since, with the reasonable assumption that the ferromagneticparamagnetic line, which is of second order, does not exhibit non-universal behaviour, we see that the gonihedric model must be in the same universality class of the ordinary three-dimensional Ising model.

The lamellar to disordered transition line turns out to be first order for $J_{4}=0$ (this being indipendently confirmed by the numerical simulations in [13]), and hence the correct phase diagram exhibit a critical end point structure, instead of the bicritical one predicted by the mean field approximation. Nevertheless, CVM results for the critical amplitude of the Ising transition close to $J_{2} / J_{1}=-1 / 4$, analyzed with Padè approximants 114. show the existence of strong cross-over effects [10], probably due to the vicinity of the lamellar spinodal line, which should be responsible for the contrasting results for the critical exponents found in the simulations [6,8]. Other previous simulations [13] were also not able to give definitive estimates of critical exponents.
At $J_{2} / J_{1}=-1 / 4$ the critical value of $1 /\left(\beta J_{1}\right)$ is 1.171. It can also be shown by CVM calculations that for other values of $J_{4}$ the ferromagneticparamagnetic transition becomes of first order close to the lamellar transition with a tricritical point at $J_{2}<0$. In the $k$-parametrization the tricritical point is observed for $k<0.86$.

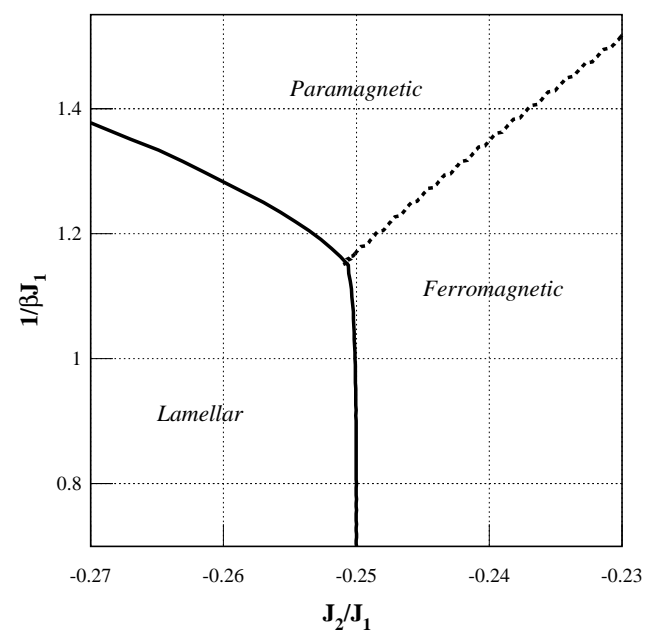

Figure 1. The phase diagram of model (1) at $J_{4}=0$. Solid and dashed lines represent first and second order transitions respectively.

In order to understand the source of the discrepancies between the various simulations, we tried also to perform a standard Metropolis Monte Carlo on this model. In Fig. 2 we give our results for the behaviour of the specific heat. Our data have been obtained by averaging over 2000 decorrelated measures at each value of the lattice size $L$ and of the inverse temperature $\beta$. Our decorrelation times were 1000 full updates of the lattice in the case of the largest value of $L$. These times are substantially longer than those reported in 6,88], but we found them necessary (at least with $L=18$ ) in order to get stable averages. This suggests that the simulations performed in [8] with much larger lattices are definitely too short.

We fitted our data with the function $C(\beta)=$ $C_{\max } /\left[b\left(\beta-\beta_{c}\right)^{2}+1\right]$ and the values of $C_{\max }$ and $\beta_{c}$ obtained for each lattice size are listed in Table 1. A fit of the position of the peak with the 
function $\beta_{c}=\beta_{\text {crit }}+a L^{-\frac{1}{\nu}}$ gives the estimate for the critical inverse temperature $\beta_{\text {crit }}=0.4370 \pm$ 0.0001 corresponding to $1 /\left(\beta J_{1}\right)=1.144$ to be compared with the CVM value 1.171 and with 1.136 of the previous simulations [6]. Our estimate for the exponent $1 / \nu$ is $1 / \nu=1.483 \pm 0.001$. Since the Ising value is $1 / \nu=1.594 \pm 0.004$ [15] we have to go to much larger lattices in order to get reliable estimates.

\begin{tabular}{ccc}
\hline \hline$L$ & $C_{\max }$ & $\beta_{c}$ \\
\hline \hline 8 & $2.8041 \pm 0.0237$ & $0.41182 \pm 0.00021$ \\
10 & $4.3707 \pm 0.0706$ & $0.41872 \pm 0.00018$ \\
12 & $6.1137 \pm 0.0334$ & $0.42300 \pm 0.00010$ \\
16 & $9.4068 \pm 0.0586$ & $0.42794 \pm 0.00004$ \\
18 & $10.6710 \pm 0.0883$ & $0.42937 \pm 0.00002$ \\
\hline \hline
\end{tabular}

Table 1

Valus of $C_{\max }$ and $\beta_{c}$ for different choices of the lattice size $L$ obtained by fitting data in Fig. 2.

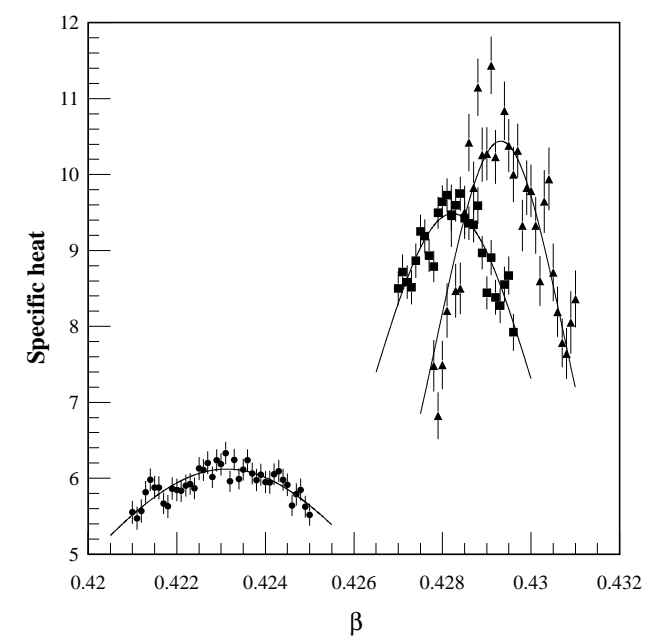

Figure 2. Monte Carlo measurements of specific heat in the gonihedric model with $\kappa=1$. Circles, squares and triangles refer respectively to $L=$ $12,16,18$. Solid lines represent the best fit of the peaks performed with the function given in the main text.

In summary, we have shown, studying the phase diagram of the model in an enlarged pa- rameter space by means of the cluster variation method, that the three dimensional gonihedric model is in the same universality class of the ordinary three dimensional Ising model. Furthermore, the model seems to exhibit very strong crossover effects which pose serious problems to numerical simulations.

We are indebted to Marcia Barbosa, Amos Maritan, Michele Caselle, Dino Cosmai and Paolo Cea for helpful discussions about this work.

\section{REFERENCES}

1. G. Gonnella, S. Lise, A. Maritan, Europhys. Lett. 32 (1995) 735.

2. See, e.g., Statistical Mechanics of Membranes and Surfaces, edited by D.R. Nelson, T. Piran, S. Weinberg, (World Scientific, Singapore, 1989).

3. G.K. Savvidy, F.J. Wegner, Nucl. Phys. B413, 605 (1994).

4. A. Cappi, P. Colangelo, G. Gonnella, A. Maritan, Nucl. Phys. B370 (1992) 659.

5. G.K.Savvidy, K.G.Savvidy and P.G.Savvidy, Phys. Lett. A 221 (1996) 233.

6. D.A. Johnston and R.P.K.C. Malmini, Phys. Lett. B378 (1996) 87.

7. R. Pietig, F. Wegner, Nucl. Phys. B466 (1996) 513.

8. M. Baig et al., preprint hep-lat/9703008

9. E.N.M. Cirillo, G. Gonnella, D.A. Johnston, A. Pelizzola, Phys. Lett. A 226, (1997) 59.

10. E.N.M. Cirillo, G. Gonnella, A. Pelizzola, Phys. Rev. E 55 (1997) R17.

11. R. Kikuchi, Phys. Rev. 81 (1951) 988; G. An, J. Stat. Phys. 52 (1988) 727.

12. R. Pietig, $\mathrm{PhD}$ thesis, Univeristy of Heidelberg (1997).

13. R.K. Heilmann et al., in Computer Simulation Studies in Condensed Matter Physics $I V$, eds. D.P. Landau, K.K. Mon and H.B. Schüttler, Springer Proceedings in Physics vol. 72 (Springer, Berlin 1993).

14. A. Pelizzola, Phys. Rev. E 53 (1996) 5825 and refs. therein.

15. A.M. Ferrenberg and D.P. Landau, Phys. Rev. B 44 (1991) 5081. 


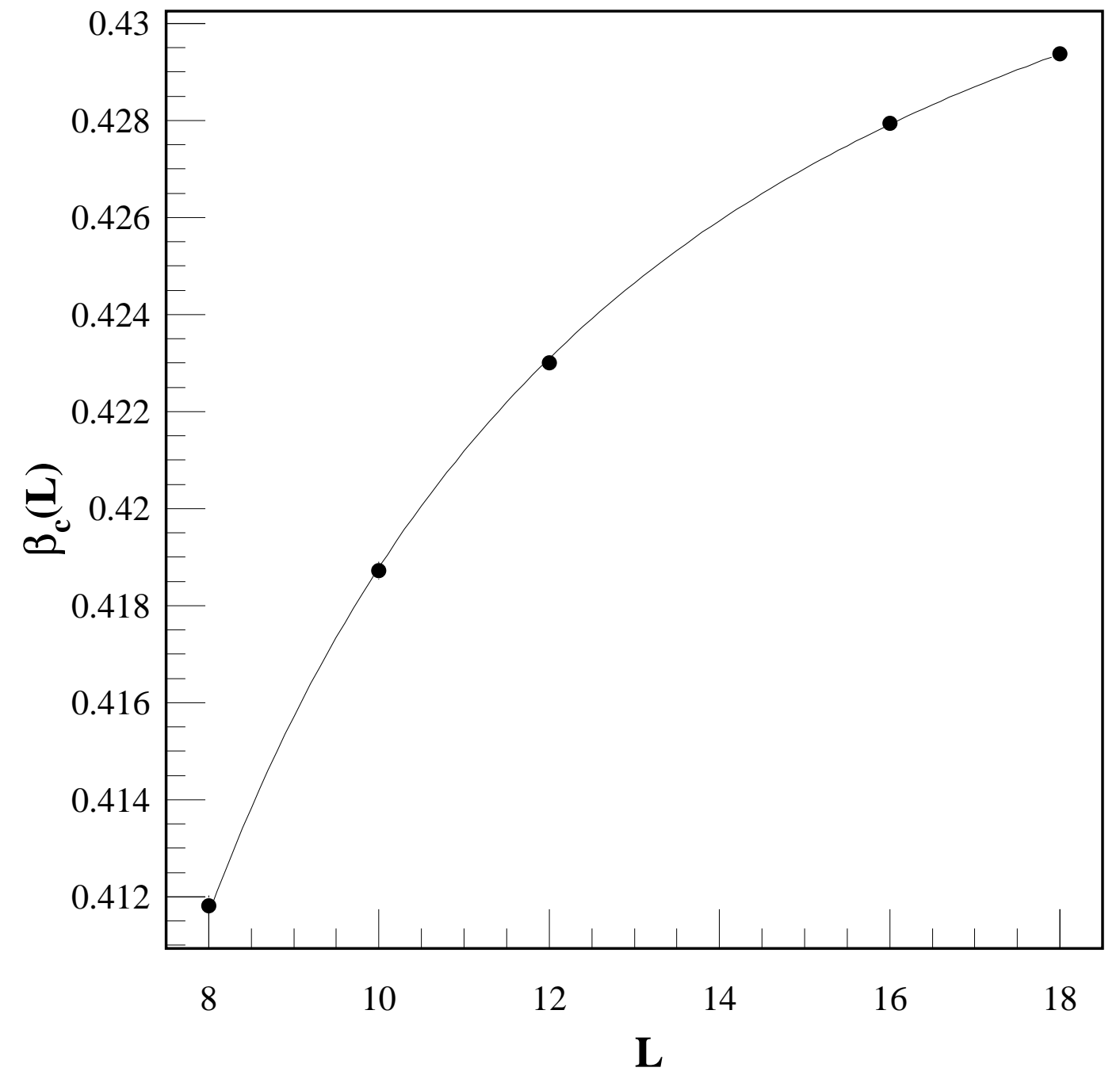

